\title{
Enantioselective Oxidation of Amines on a Gold Electrode Modified by a Self-Assembled Monolayer of a Chiral Nitroxyl Radical Compound
}

\author{
Yoshitomo Kashiwagi ${ }^{\dagger}$, Kazumi UchiYama, Futoshi Kurashima, Jun-ichi AnzaI and Tetsuo Osa \\ Graduate School of Pharmaceutical Sciences, Tohoku University, Aobayama, Aoba-ku, Sendai 980-8578, Japan
}

Keywords Self-assembled monolayer, electrocatalytic oxidation, nitroxyl radical

Organic nitroxyl radicals, such as 2,2,6,6-tetramethylpiperidin- $N$-oxyl (TEMPO), are known to be an effective redox mediator for the oxidation of amines. ${ }^{1,2}$ We have reported that a graphite felt electrode coated with a thin poly(acrylic acid) layer immobilizing 4amino-TEMPO can be used for the electrocatalytic oxidation of amines. ${ }^{3}$ On the other hand, a self-assembled monolayer has attracted a great deal of interest because of its possible applications to many fields, such as biosensors and display devices. ${ }^{4,5}$ Many self-assembled monolayer-modified electrodes report on the monolayer-immobilization of thiols containing redox active functional groups on gold electrodes..$^{6-10}$ Recently, Fuchigami et al. ${ }^{11}$ have prepared a self-assembled TEMPO-modified electrode for the electrocatalysis of 4-methylbenzyl alcohol.

In this pepar, we report on the enantioselective voltammetric behavior of chiral amines at gold electrodes modified with a mixed monolayer of chiral nitroxyl radical compound and hexadecanethiol.

\section{Experimental}

\section{Reagents}

$(6 S, 7 R, 10 R)$ - $N$-Oxyl-2,2,7-trimethyl-10-isopropyl-1azaspiro[5.5]-undecane-1,2-dithiolane-3-pentanecarboxamide (1) or $\mathrm{N}$-oxyl-2,2,6,6-tetramethylpiperidin1,2-dithiolane-3-pentanecarboxamide (2) was synthesized by the reaction of $(6 S, 7 R, 10 R)-4$-amino-2,2,7trimethyl-10-isopropyl-1-azaspiro[5.5] undecane-1yloxyl $^{12}$ or 4-amino-2,2,6,6-tetramethylpiperidin-1yloxyl (4-amino-TEMPO), respectively, with D,L- $\alpha$ lipoic acid in the presence of $N, N$-dicyclohexylcarbodiimide. Hexadecanethiol (Tokyo Chemical Industry) was used as received. Acetonitrile $\left(\mathrm{CH}_{3} \mathrm{CN}\right.$, guaranteed reagent, Nakarai Tesque Inc.) was stored over molecular sieves $(4 \AA, 1 / 16)$ for $24 \mathrm{~h}$ and then distilled in the presence of $\mathrm{CaCl}_{2}$. Sodium perchlorate $\left(\mathrm{NaClO}_{4}\right.$, guaranteed reagent, Nakarai Tesque Inc.) was recrystallized from $\mathrm{CH}_{3} \mathrm{OH}$. All other reagents used as sub-

\footnotetext{
† To whom correspondence should be addressed.
}

strates of electrocatalytic oxidation were of commercially available reagent grade.

\section{Preparation of modified electrode}

A gold ( $\mathrm{Au})$ disk electrode (1.6 $\mathrm{mm}$ diameter) was briefly etched in a 1:3:4 (in volume) mixture of conc. $\mathrm{HNO}_{3} / \mathrm{conc}$. $\mathrm{HCl} /$ water before use. For preparing selfassembled monolayers on the surface of a Au electrode, the Au electrode was immersed in an ethanol solution containing a mixture of $1 \mathrm{mM} 1$ or 2 and $0.05 \mathrm{mM}$ hexadecanethiol. The electrode was treated in the solution for at least $1 \mathrm{~h}$ and rinsed with ethanol and water. In the present study, mixed monolayers of $\mathbf{1}$ and $\mathbf{2}$ with hexadecanethiol (20:1 by mol) were used because the pure monolayer was reported to be somewhat unstable during electrochemical measurements. ${ }^{11}$

\section{Apparatus and procedure}

Cyclic voltammetry was carried out in a $\mathrm{CH}_{3} \mathrm{CN}$ solution containing $0.1 \mathrm{M} \mathrm{NaClO}_{4}$ as a supporting electrolyte and $80 \mathrm{mM}$ 2,6-lutidine as a deprotonating agent. The modified electrode was employed as a working electrode, and a platinum wire was used as a counter electrode. The anode potentials were referred to $\mathrm{Ag} / \mathrm{AgCl}\left(0.1 \mathrm{M} \mathrm{NaClO}_{4}\right.$ in $\left.\mathrm{CH}_{3} \mathrm{CN}\right)$. Cyclic potential sweeps were generated by a Hokuto Denko Model HAB-151 potentiostat/galvanostat. Cyclic voltammograms were recorded on a Graphtec Model WX1200 XY recorder. All electrochemical measurements were carried out at room temperature.

\section{Results and Discussion}

Figure 1 shows the cyclic voltammograms (CVs) of the mixed monolayer containing $\mathbf{1}$ or $\mathbf{2}$ formed on the Au electrode. Both electrodes exhibited symmetrical reversible redox waves characteristic of a surface-confined species undergoing reversible redox reactions. The redox potential of $\mathbf{1}$ and $\mathbf{2}$ monolayer-modified electrodes was $+0.62 \mathrm{~V}$ and $+0.60 \mathrm{~V} v s$. $\mathrm{Ag} / \mathrm{AgCl}$, respectively. The peak split between the anodic and cathodic peak potentials was $c a .20 \mathrm{mV}$ and a linear 
relationships was observed between the anodic and cathodic current $\left(i_{\mathrm{pa}}\right.$ and $\left.i_{\mathrm{pc}}\right)$ and potential scan rate ranging from 10 to $200 \mathrm{mV} \mathrm{s}^{-1}$. The amount of immobilized $\mathbf{1}$ and $\mathbf{2}$, as determined by integration of current peak of the CVs, was $3.4 \times 10^{-10}$ and $5.7 \times 10^{-10} \mathrm{~mol} \mathrm{dm}^{-2}$, respectively. The surface coverage of $\mathbf{1}$ was slightly less than 2, a monolayer-modified electrode, probably due to the bulkier nature of $\mathbf{1}$ than 2. Both of the nitroxyl redical monolayer-modified electrodes were quite stable, and no decay in the CVs was observed after potential scanning many times.

The electrocatalytic activity of the monolayer-modified electrodes towards the oxidation of chiral amines was investigated. Figure 2 shows the CVs of $10 \mathrm{mM}$ $(R)-(+)$ - and $(S)-(-)-1$-phenylethylamine measured on the nitroxyl radical monolayer-modified electrodes in the presence of $80 \mathrm{mM}$ 2,6-lutidine as a deprotonating agent. When a self-assembled chiral 1 monolayermodified electrode was used, the anodic peak for the oxidation of $(R)-(-)$-1-phenylethylamine was slightly lager than that for a blank (the electrode itself). On the contrary, the anodic peak for the oxidation of $(S)-(-)-1-$ phenylethylamine was highly enhanced, and no cathodic peak was observed on the reverse scan, showing
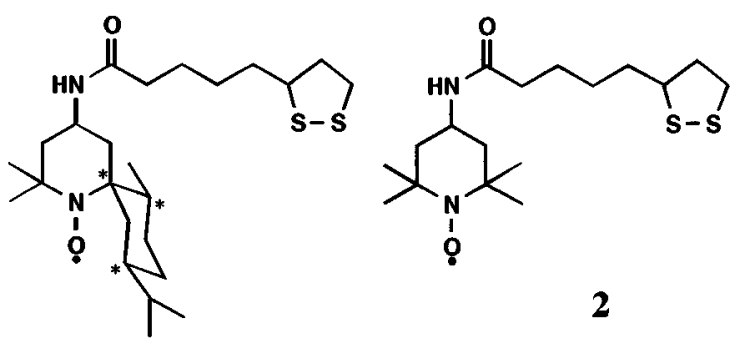

1

Chart 1 Structures of $\mathbf{1}$ and $\mathbf{2}$. that $(S)-(-)-1-$ phenylethylamine was oxidized into the imine form electrocatalytically. ${ }^{1}$ In other words, the $\mathbf{1}$ monolayer-modified electrode discriminates $(R)$ - and $(S)$-isomer in the electrocatalytic oxidation reaction. The direct oxidation of both $(R)-(+)-$ and $(S)-(-)-1$ phenylethylamine was observed to be more positive at $+0.8 \mathrm{~V} v$ s. $\mathrm{Ag} / \mathrm{AgCl}$, which is an enormous increase in the anodic current. The achiral 2 monolayer-modified electrode, on the other hand, exhibited a large electrocatalytic wave for both $(R)-(+)-$ and $(S)-(-)-1-$ phenylethylamine along with the disappearance of the cathodic peak. This means that monolayer-modified electrode $\mathbf{2}$ is electrocatalytically active equally for the

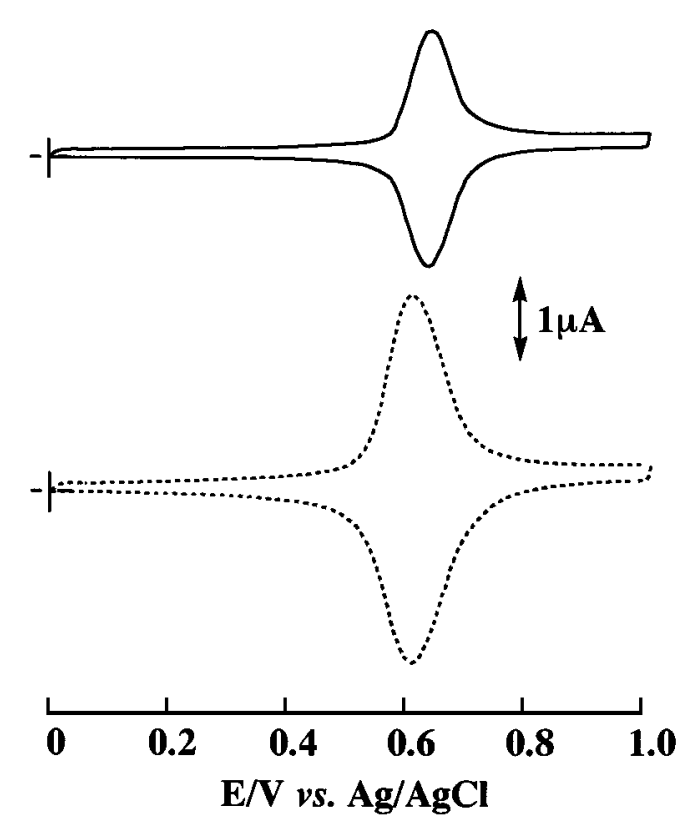

Fig. 1 Cyclic voltammograms of $\mathbf{1}(-)$ and 2 (---) monolayer-modified electrode in $0.1 \mathrm{M} \mathrm{NaClO}_{4} / \mathrm{CH}_{3} \mathrm{CN}$ at the scan rate of $100 \mathrm{mV} / \mathrm{s}$.

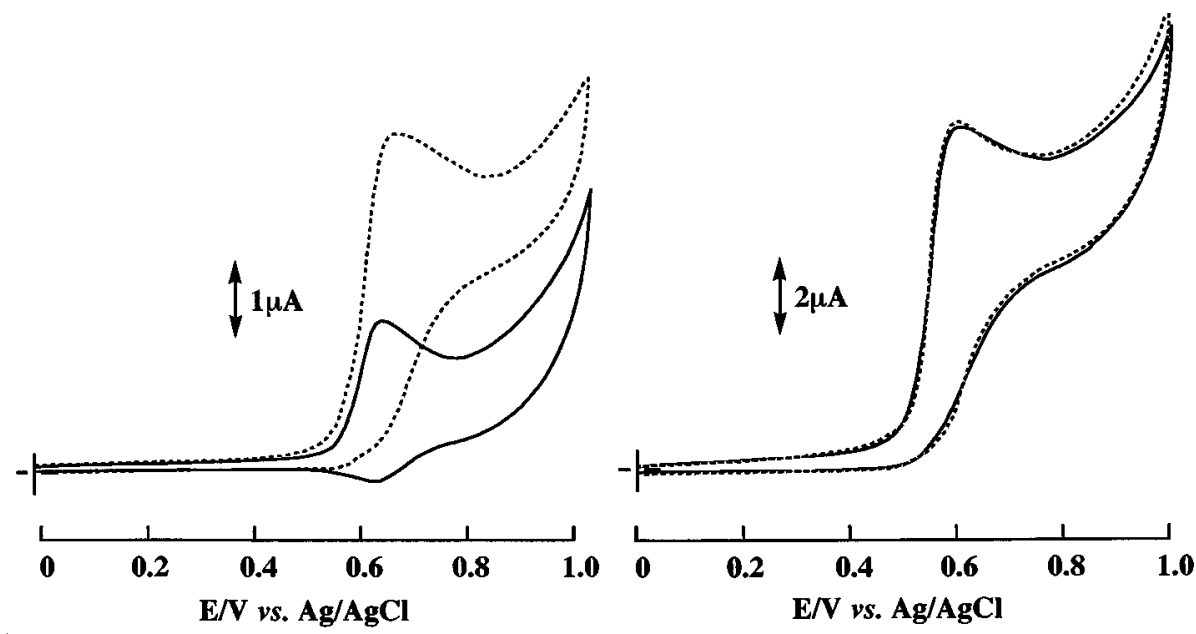

Fig. 2 Cyclic voltammograms of $\mathbf{1}$ (left) and $\mathbf{2}$ (right) monolayer-modified electrode in $0.1 \mathrm{M} \mathrm{NaClO}_{4} / \mathrm{CH}_{3} \mathrm{CN}$ at the scan rate of $100 \mathrm{mV} / \mathrm{s}$. - : in the presence of $10 \mathrm{mM}(R)-(+)-1$-phenylethylamine and $80 \mathrm{mM}$ 2,6-lutidine; ---: in the presence of $10 \mathrm{mM}(S)-(-)-1$-phenylethylamine and $80 \mathrm{mM}$ 2,6-lutidine. 
Table 1 Catalytic efficiency of $\mathbf{1}$ monolayer-modified electrode for the oxidation of chiral amines

\begin{tabular}{lccc}
\hline Substrate & Config. & $i_{\text {cat }} / \mathrm{mA}$ & Ratio $^{\mathrm{a}}$ \\
\hline & $R$ & 1.37 & 2.24 \\
& $R$ & 3.07 & \\
& $S$ & 1.44 & 2.98 \\
& $R$ & 1.42 & \\
& $S$ & 3.76 & \\
& $R$ & 6.00 & 1.00 \\
& $S$ & 5.97 & \\
\hline $\mathrm{NH}_{\mathrm{NH}_{2}}$ & $S$ & &
\end{tabular}

a. $i_{\text {cat. }}$ of $S / i_{\text {cat. }}$ of $R$.

oxidation of both $(R)-(+)-$ and $(S)-(-)-1$-phenylethylamine.

Table 1 gives the catalytic efficiency of the monolayer-modified electrodes $\mathbf{1}$ for the oxidation of several chiral amines. The ratios between $R-i_{\text {cat. }}$ (catalytic peak current $\left(i_{\text {cat. }}\right)$ for the oxidation of $R$-isomer) and $S-i_{\text {cat }}$ $\left(i_{\text {cat. }}\right.$ for the oxidation of $S$-isomer) of 1-phenylethylamine, 1-(1-naphthyl)ethylamine and 1-cyclohexylethylamine, which has a chiral center at the $\alpha$-position to the amino group, were $2.98,2.65$ and 2.24 , respectively. This results suggest that the electrocatalytic oxidation of $S$-isomers on a monolayer-modified electrode 1 , was prior to $R$-isomers. On the contrary, the ratio between $R-i_{\text {cat }}$ and $S$ - $i_{\text {cat. }}$ of 2-phenylpropylamine, which has a chiral center at the $\beta$-position to the amino group, was unity, which suggests no enantioselectivity of the electrode to 2-phenylpropylamine. These facts mean that an $\alpha$-hydrogen of the chiral center adjacent to the amino group will be necessary to attain enantioselective oxidation in the present electrochemical method. On the other hand, the ratio between $i_{\text {cat }}$ for the oxidation of $R$-isomer and $S$-isomer in all chiral amines by the monolayer-modified electrode 2 was unity. This results suggest that the monolayer-modified electrode 2 afforded no enantioselectivity for chiral amines. The enantioselective oxidation on the electrode modified with the chiral nitroxyl radical-terminated self-assembled monolayer may be applied to the determination of optical purity of chiral amines.

This work was supported in part by Grants-in-Aid for Scientific Research on Priority Areas (No. 10132206: "Innovative Synthetic Reactions") and for Encouragement Research (No. 10771266) from the Ministry of Education, Science, Sports and Culture of Japan.

\section{References}

1. M. F. Semmelhack and C. R. Schmid, J. Am. Chem. Soc., 105, 6732 (1983).

2. F. MacCorquodale, J. A. Crayston, J. C. Walton and D. J. Worsfold, Tetrahedron Lett., 31, 771 (1990).

3. Y. Kashiwagi, F. Kurashima, C. Kikuchi, J. Anzai, T. Osa and J. M. Bobbitt, J. Chin. Chem. Soc., 45, 135 (1998).

4. K. R. Stewart, G. M. Whitesides, H. P. Grodfried and I. F. Silvera, Rev. Sci. Instrum., 57, 1381 (1986)

5. J. A. M. Sondag-Huethorst and L. G. J. Fokkink, Langmuir, 10, 4380 (1994).

6. K. Uosaki, Y. Sato and H. Kita, Langmuir, 7, 1510 (1991).

7. J. Li and A. E. Kaifer, Langmuir, 9, 591 (1993).

8. L. Zhang, T. Lu, G. W. Gokel and A. E. Kaifer, Langmuir, 9, 786 (1993).

9. Y. Sato and K. Uosaki, Denki Kagaku (presently Electrochemistry), 61, 816 (1993).

10. K. V. Gobi, K. Tokuda and T. Ohsaka, Denki Kagaku (presently Electrochemistry), 65, 498 (1997).

11. T. Fuchigami, T. Shintani, A. Konno, S. Higashiya and T. Nonaka, Denki Kagaku (presently Electrochemistry), 65, 506 (1997).

12. Z. Ma, G. Huang and J. M. Bobbitt, J. Org. Chem., 58, 4837 (1993).

(Received April 30, 1999)

(Accepted May 31, 1999) 